\title{
Research on the Influence of Internet Word of Mouth on Consumers' Purchase Intention
}

\author{
Jun Wang ${ }^{1, a *}$ and Hai Liu ${ }^{2, b}$
}

${ }^{1}$ No.3, huangjiahu west road, hongshan district, wuhan city, hubei province, China,Wuhan Technology and Business University Hubei Business Service Research Development Center;

${ }^{2}$ No.3, 1863, liberty avenue, wuhan, hubei province, China, Yangtze river water conservancy commission.

a1075045780@qq.com; b8930524@qq.com.

Keywords: IWOM; Purchase intention; Purchase behavior; Purchase decision-making

\begin{abstract}
With the development of information technology, more and more consumers tend to use the network platform to release or search enterprise products, brands, services and so on. Under this circumstance, this paper selected the influence of Internet word of mouth (IWOM) on consumers' purchase intention as a research focus. Based on four aspects, i.e. word-of-mouth related concept, purchase intention, influence mechanism of IWOM communication, and consumers' purchasing decision-making process model, this paper made a systematic review on a lot of scholars' studies on the influence of IWOM on consumers' purchase intention.
\end{abstract}

\section{Word of Mouth Related Concepts}

Traditional Word of Mouth. Word-of-mouth is the earliest way of communication between people every day. Researches on word of mouth emerged in the 1950s or 1960s, Chinese scholars Huang Ying, Zhu Shunde also ever made definitions for word of mouth. At present, the results of word of mouth researches are very rich and the mainstream definitions have been listed by the author in Table 1.

Table 1 Definition of traditional word of mouth

\begin{tabular}{|c|c|}
\hline Chinese and foreign scholars & Definitions of traditional word of mouth \\
\hline Arndt & $\begin{array}{c}\text { Informal, face-to-face and oral } \\
\text { communication between two } \\
\text { non-commercial parties on brand, product } \\
\text { or service [1]. }\end{array}$ \\
\hline Huang Ying, Zhu Shunde & $\begin{array}{l}\text { A kind of information disseminated by a } \\
\text { third party other than merchants and } \\
\text { consumers through a certain way on a } \\
\text { particular product, service or brand [2]. }\end{array}$ \\
\hline Huang Xiaojun, Xu Weiqing & $\begin{array}{l}\text { Word of mouth communication refers to } \\
\text { the informal interpersonal } \\
\text { communication behavior between two } \\
\text { non-commercial parities on a product, } \\
\text { brand, organization and service [3]. }\end{array}$ \\
\hline
\end{tabular}

It can be seen that the definition of traditional word of mouth includes the following three aspects: first, the traditional word of mouth information contains products, services and brands, etc.; second, the traditional word of mouth has positive and negative aspects; third, the word of mouth will affect the consumers' purchasing decision and purchasing determination to varying degrees.

IWOM. With the rapid development of information technology, more and more consumers are used to use the network platform to publish or search for enterprise products, brands, services and so on. In this paper, Dong Dahai's definition for IWOM is applied that IWOM is a kind of 
bidirectionally interactive, non-commercial communication between consumers with strong relationship via the medium of text [4]. It can be seen that the definition of IWOM includes the following four aspects: first, IWOM information contains products, services and brands, etc.; second, IWOM has its positive and negative aspects; third, IWOM will affect consumers' purchase decision and purchasing determination to varying degrees; fourth, the IWOM mainly conveys consumers' evaluation on products, services and brands through the Internet platform.

The Differences Between IWOM and Traditional Word of Mouth. Both IWOM and traditional word of mouth convey consumers' expression of various types of information and ideas toward enterprise products or services, but due to the special nature of the network platform, there are differences between these two types of word of mouth. Compared to traditional word of mouth, IWOM mainly has differences reflected in the following four aspects:

Anonymity.The information dissemination on the network platform is carried out in an anonymous form, and the information receiver is generally difficult to know the true identity of the publishers. Because the information publishers can express with an anonymous and unknown identity, they are more willing to provide real purchase experience and view on the products, and they are also willing to communicate and share with others no matter they have positive attitude or negative attitude toward products. The anonymity of IWOM makes the publishers and communicators face less pressure from public opinions and can protect their personal privacy. Consumers can freely express their opinions and express real feelings on the web platform, in addition, with the help of network platform, the communication between consumers will be more open and extensive.

Wide range.Information expressed by consumers on the network platform can be copied and disseminated for many times. Most of the traditional word of mouth is disseminated in the private circle, the scope of the dissemination is among acquaintances of consumers, while the dissemination on network platform has a wider range, the unfamiliar strangers can be the dissemination audiences, and its dissemination speed is faster and more efficient.

Non-face-to-face communication.IWOM is disseminated by synchronous or asynchronous communication on the Internet platform, while the traditional word of mouth mainly relies on face-to-face communication by interpersonal relationships. Non-face-to-face communication form of IWOM will make communication faster and more effective, and will also reduce the impact of the objective environment. As long as consumers have a terminal that can connect to the Internet, they can share and release information. The traditional word of mouth is disseminated in a one-to-one communication mode, and the IWOM can be disseminated in one-to-one communication mode or one-to-many communication mode.

Information tangibility.The traditional word of mouth is mainly disseminated in the form of oral language, but this information may be partly missing after dissemination and is thereby difficult to be completely preserved, its essence can not be accurately grasped and understood. IWOM information can be preserved via texts, pictures, videos and other forms, so that consumers can repeatedly analyze and accurately grasp the information, such feature both extends the life time of disseminated information and strengthens its influence. Some studies also found that text information is more logical than face to face information exchange. The intangible characteristics of the spread of IWOM provide researchers a lot of information sources for reference and also promote the development of IWOM marketing.

\section{Purchase Intention}

Definition of Purchase Intention. Purchase intention refers to the subjective tendency of consumers to choose a brand or product, and the purchase intention represents the subjective probability of consumers for buying a product or service [5].

Definition of Purchase Behavior. Purchase behavior refers to a kind of behavior in which consumers' searching, purchasing, evaluation and handling of products, services or ideas in order to meet the needs [6]. Meeting the own needs of consumers is the main purpose of purchase behavior, with such purpose they participate in consumer activities and conduct purchase behavior. 
The Relationship Between Purchase Intention and Purchase Behavior. This study also focuses on whether consumers' purchase behavior can be predicted by the purchase intention of consumers, or whether purchase intention can be adopted by consumers to provide a reference for the purchase behavior. The study found that purchase behavior can be predicted by consumers' purchase intention. IWOM still has an impact on offline purchases. TRA hypothesis suggested that people's behavior is rational, and according to the measurement based on attitudes, beliefs and intentions, the intentional behavior is based on psychological activities [7], as shown in Figure 1:

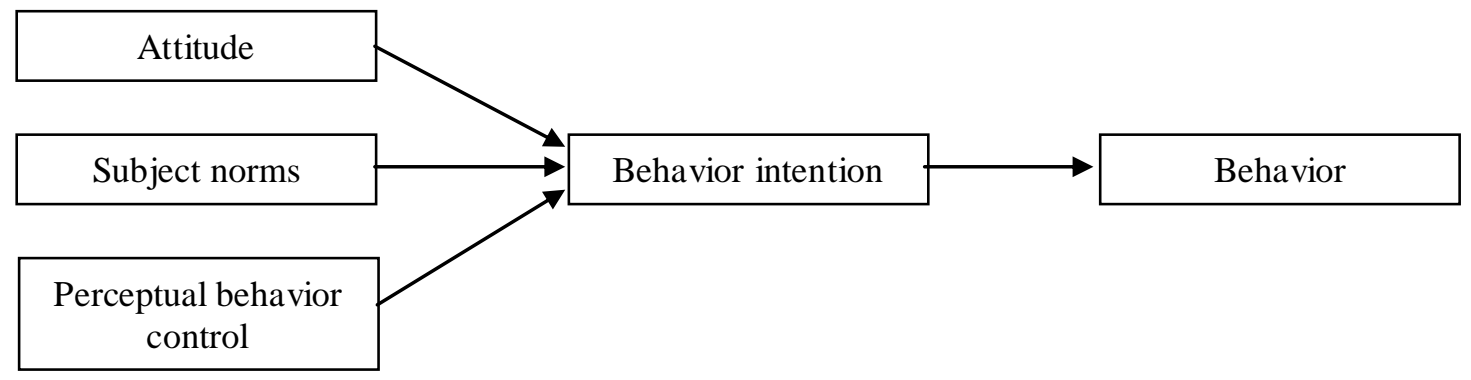

Fig 1 TRA model

\section{The Influence Mechanism of IWOM Dissemination}

From the existing literatures, the main factors influencing the spread of word of mouth are shown as the following: IWOM's features, senders, receivers, the relationship between sender and receiver of information.

IWOM's Features. The features of IWOM mainly include the following four aspects: the information quality of IWOM, the quantity of IWOM, the form of IWOM, the timeliness of IWOM [8].

The quality of IWOM refers to whether the IWOM has real objectivity, whether it is easy to understand and related to the products. The quantity of IWOM refers to the quantity of product comments. The higher the quantity is, the more intensively the product is favored by consumer. The form of IWOM is mainly divided into subjective and objective forms, the objective form is mainly reflected in comments for the characteristics of the product; the subjective form refers to the customers' feelings and emotional expression after purchasing products or services. The timeliness of IWOM refers to whether the comments on the product are up-to-date, timely and effective and whether the demand is met [9].

IWOM Senders. This factor of IWOM mainly concerns about the reliability of websites and publishers. The sender sends the word of mouth information through the website media, while the receiver also obtains product and service evaluation through the website. On the IWOM, the higher the reliability of the site is, the greater the impact of IWOM is.

IWOM Receivers. With difference in the ability and experience in word of mouth receivers, the influence of the same word-of-mouth information on different receivers varies. In general, receivers will take the initiative to use the network platform to search for information, then word-of-mouth communication will be carried out.

According to the study, when the word of mouth receivers have a perceived risk before the purchase of products, they will search for product information. Product involvement, individual personality and professional knowledge are main factors that affect the receiver's ability [9].

The theory of product involvement suggests that a high degree of involvement can make consumers difficult to accept comments that differ from their opinions, in contrast, the comments similar to their own opinions are often easily accepted [10]. The consumer's network involvement is about the initiative of consumers using the web for information search and their familiarity with the network. In other words, the deeper the degree of consumer's network involvement is and the more familiar the network operation is, the more accurate the judgment on IWOM information is, thus good results may be achieved. 
Perceive D Risk. Perceived risk shows the uncertainty of the purchase behavior before the purchase decision is made, and people often want to use the IWOM information to reduce the perceived risk of purchasing the product.

Anne Sophie Cases argues that online shopping includes eight perceived risks, namely, risks about finance, time, delivery, payment, performance, society and source [11].

Trust. Psychology theories believe that trust is an irrational act, and trust comes from the reliability evaluated by individuals or groups on commitment, verbal or written statements [12].

\section{Consumers' Purchase Decision-making Process Model}

Howard-Sheth Model. Howard-Sheth model was proposed by the scholar Howard in 1963, after the cooperation with Sheth, it was formally formed in 1969 after the revision. It focuses on four aspects, i.e. stimulus factor, external factor, internal factor, response factor, for a comprehensive analysis of consumers' purchasing decision-making behavior. The Howard-Sheth model comes from the "stimulus-response" concept. The whole model consists of three parts: inputs, the consumers are allowed to receive information by external stimuli from three types of stimulus sources: physical stimulation, symbolic stimulus and social environment stimulus; perception and learning construction, this part mainly describes how consumers deal with the impression formed in the brain after obtaining stimulus information, and their purchase intention are produced based consumer's own motivation, confidence and other factors; outputs, after the above stimulus, cognitive and learning reactions, the final result is the purchase behavior, including attention, brand awareness, attitude, intention and purchase behavior.

Howard-Seth model argues that input factor and external factor are the stimulus for purchasing, which can evoke and form motivations and provide the information about a variety of options, thereby affecting the customers' mental activity (internal factor). Consumers are stimulated by the stimulus and the experience of the previous purchase and begin to receive information and produce a variety of motivations, produce a series of reactions for product selection and form a series of purchasing decision-making intermediary factors, such as the selection of evaluation criteria and intentions. Under the interaction among motives, buying options and mediating factors, it will produce a certain tendency and attitude. Such tendency or attitude will lead to purchase results after combination with restriction of purchasing behavior. The feelings formed from the purchase results will be fed back to the consumer, affecting the psychology of consumers and the next purchase behavior.

Nicosia Model. Nicosia in 1966 in the book "Consumers' Decision-making Process" put forward a decision-making model: Nicosia model. This model mainly divides consumers' purchase process into a flow chart of decision-making process in order to simulate the consumers' decision-making process.

The model has four major parts:

First part, it covers from information sources to consumer attitudes and includes both enterprise and consumer's attitudes. Information flow: manufacturers transmit the product-related information to consumers through advertising and other media, through the consumers' internalization, attitudes are formed.

Second part, consumers investigate and evaluate the goods and form the output of the purchase motives. Information seeking and program evaluation: after the formation of consumer attitudes, they begin to show interest in manufacturers' products and take information collection as assessment criteria, resulting in purchase motives.

Third part, consumers take effective decision-making behavior. Purchasing behavior: consumers transform motivations into the actual purchase action, this process may be influenced by the brand availability and dealer factors.

Fourth part, the results of consumers' purchase behavior are memorized and stored by brain for reference to consumers' later purchase decision or giving feedback to the enterprise. Information feedback: after buying the product and use process, customers have practical experience toward the purchased products, and their satisfaction after use will also influence their re-purchase behavior, in 
addition, manufacturers can also gain information feedback from the consumers' purchase intention and satisfaction, so as to get a reference basis for quality improvement, pricing, advertising, and other marketing strategies.

Engel Model. Engel model is a very complete and clear theory about consumer behavior. The model was proposed by Engel, Kollat and Blackwell in 1968 and revised to form a mature theoretical framework in 1984. Its focuses on analysis from purchase decision-making process. The whole model is divided into four parts: the central control system (i.e. the process of psychological activities of consumers), information processing, decision-making process and environment.

Engel model believes that the external information, under the actions of tangible and intangible factors, is input into the central control system, that is, the information about personal experience, evaluation criteria, attitudes and personality that can cause brains' awakening, discovery, attention, understanding, memory, storage and other activities are filtered and processed to constitute the information processing procedures, and the study, assessment and selection are made internally, then external exploration, selection or assessment were activated to produce a decision-making program. In the entire decision-making, research and evaluation process, it will also be subject to environmental factors, such as income, culture, family, social class and so on. Finally the purchase process is completed, and consumers get experience for the purchased goods and conclusion about satisfaction. This conclusion will also enter the central control system through the feedback, thereby forming information and experience and affecting the purchase behavior in the future.

Kotler Model. Philip Kotler presents a pattern of consumer behavior that emphasizes both aspects of society. The model suggested that the reaction of consumers' purchase behavior will not only be affected by marketing, but also by external factors. And consumers with different characteristics will produce different processes of psychological activities. Through consumers' decision-making process, a certain purchase decision can be formed, thereby forming the consumers' purchase decisions about products, brands, distributors, purchase time and quantity.

\section{References}

[1] Q.F. Li. Empirical study of the impact of negative word of mouth on customers' purchase decision for mobile phones [D]. Hebei: Hebei University of Technology: Hebei University of Technology, 2010.(in Chinese)

[2] Y. Huang, S.D. Zhu. Management Frontier, (2003) NO.6, p.33-36. (in Chinese)

[3] W.Q. Xu, X.J. Huang. Foreign Economy and Management, (2004) NO.26, p.26-30. (in Chinese)

[4] D.H. Dong, Y. Liu. Chinese Journal of Management,(2012) NO.27 (3), p428-436. (in Chinese)

[5] J.Y. Feng,W.S. Mu and Z.T. Fu. Modern Management , (2006) NO.40 (11), p90-99. (in Chinese)

[6] J.J. Zhang. Study on the influential factors of re-dissemination of IWOM in network environment (Zhejiang University, China,2007). (in Chinese)

[7] M. Jing, Y. Zhou. Shanghai Management Science, (2005) NO.5, p5-7. (in Chinese)

[8] Q.Q. Li. Study on the influential factors of IWOM on college students' brand attitudes (MS. South China University of Technology, China 2010). (in Chinese)

[9] Y.W. Zheng. The impact mechanism of IWOM on consumption decision-making (Shanghai Jiaotong University, China,2011). (in Chinese)

[10]Z.L. Han. Foreign Economy and Management, (1997) NO.1, p11-13,17. (in Chinese)

[11] S.S. Lin, J.H. Tang. Marketing Review, (2010) NO.7 (2), p187-208. (in Chinese)

[12]X.N. Shi. Empirical study of the impact of negative IWOM on college students' purchase decision for mobile phones (Chongqing University, China, 2012). (in Chinese) 